\title{
Development and Evaluation of a Centaur Robot
}

\author{
Satoshi Tsuda ${ }^{1}$, Kuniya Shinozaki ${ }^{1}$, and Ryohei Nakatsu ${ }^{2}$ \\ ${ }^{1}$ Kwansei Gakuin University, School of Science and Technology \\ 2-1 Gakuen, Sanda, 669-1337 Japan \\ \{amy65823, scbc0052\} @kwansei.ac.jp \\ ${ }^{2}$ National University of Singapore, Interactive \& Digital Media Institute \\ Block E3A, \#02-04, 7 Engineering Drive 1, Singapore 117574 \\ idmdiranus.edu.sg
}

\begin{abstract}
Recently various types of robots are being studied and developed, which can be classified into two groups: humanoid type and animal type. Since each group has its own merits and demerits, a new type of robot is expected to emerge with greater strengths and fewer weaknesses. We have proposed a new type of robot called the "Centaur Robot" by merging the concepts of these two types of robots. This robot has a human-like upper body and a four-legged animal-like lower body. Due to this basic architecture, we have found that the robot has several merits, including human-like behaviors and stable walking. This time we have carried out the experiments to evaluate the stability of its waling motion. This paper described the detailed results of the experiments as well as the construction of the software/hardware of the robot.
\end{abstract}

\section{Introduction}

It is expected that in the near future various kinds robots would be introduced into our home. By interacting with us using verbal/nonverbal communication functions, they would support us in our everyday life and/or entertain us. Various robots for such purpose are being studied and developed in research institutes and companies that can be classified into two groups: a humanoid robot with two legs [1][2], an animal type robot with four or more legs [3][4][5][6]. Also a humanoid robot can be classified into those with two legs and those with wheels [6]. Each of these types has its own merits. The design of a humanoid robot with two legs is based on humans and can mimic such human motions as walking. Since this robot's behavior resembles human behavior, it might easily be introduced into society. At the same time, however, its walking capability still lacks stability, and it sometimes falls down, restricting its area of activity. Also it has difficulty maintaining its balance on ground that is not flat. On the other hand, the merit of an animal type robot is its four legs, which allow it to walk stably even on uneven ground. Since it can also basically stand on three legs, it can adopt to various ground pattern changes. So far, however, the robot has mainly been developed as a pet to which useful applications have rarely been applied. A humanoid robot with wheels for locomotion, which we call a wheel type robot, can move very smoothly and stably on the ground. It rarely falls down. It can even move on slightly uneven ground. On the other hand, it has no ability to move on stairs, which greatly restricts its area of activity since houses usually contain stairs and other types of height differences. 
In the previous paper, we have proposed an approach to overcome these problems. We proposed a new type of robot with a human-like upper body and an animal-like lower body that we call a "Centaur Robot [7]." In the previous paper, we described its basic concept and then its detailed software/hardware architectures. Also we pointed out that the robot could achieve stable walking motions. This time we carried out several experiments to quantitatively evaluate the stability of walking. In this paper we first simply introduce the software/hardware construction of the robot. Then we will describe the details of the experiments.

\section{Related Works}

Recently, especially in Japan, various kinds of robots have been studied and developed, particularly humanoid robots that are expected to support our daily life. For example, HONDA has developed a humanoid robot called ASIMO that has sophisticated walking capability [1]. For animal types of robots, on the other hand, most have been studied and developed as pets instead of supportive robots, including AIBO developed by Sony [3].

Although much research/development continues on humanoid and animal types of robots, little research has integrated these two types for several reasons. One reason is that since there are so many research themes for new functions and improvements for each of these types of robots, researchers have little incentive to concentrate on new types of robots that go beyond humanoid or animal types. Another is that even myths or folktales only contain a few examples of such creatures as centaurs, mermaids, and sphinxes in which humans and animals are integrated. Thus it is rather hard to imagine the functions and application areas that such a new type of robot might have.

Taking these situations into considerations, we have proposed and developed a centaur robot because we believed by integrating two types of robots we could create a new type of robot with advantages over conventional robots [7]. In the previous paper, we indicated that the robot has a capability of stable walking motions. In this paper we will describe the detailed results of the walking stability evaluation.

\section{Humanoid Robots}

In our work, we are developing a robot that can stably achieve various motions by merging two types of robots: a humanoid and an animal.

There are two approaches for such integration: from the humanoid robot side and from the animal robot side. The former approach tries to realize a four-legged body as well as four-legged walk while maintaining a human-like upper body and achieving human-like motions. On the other hand, the latter approach achieves various humanlike motions by adding a human upper body to a four-legged robot. In our study, we chose the former approach and modified the hardware and software of a humanoid robot to realize a centaur robot.

We adopted a humanoid robot developed by Nirvana Technology as a platform robot [8][9][10]. This robot has 22 servo motors that can express various human-like motions. Figure 1 shows its appearance, and Table 1 shows its specifications. Figure 2 illustrates the construction of its hardware. The control board, on which a 
microprocessor $\mathrm{SH} 2$ is attached, is connected to the servo motors, a gyro sensor, acceleration sensors, PC, and a battery. The program on the controller can achieve autonomous robot behaviors. At the same time, we can send commands to the robot by PC.

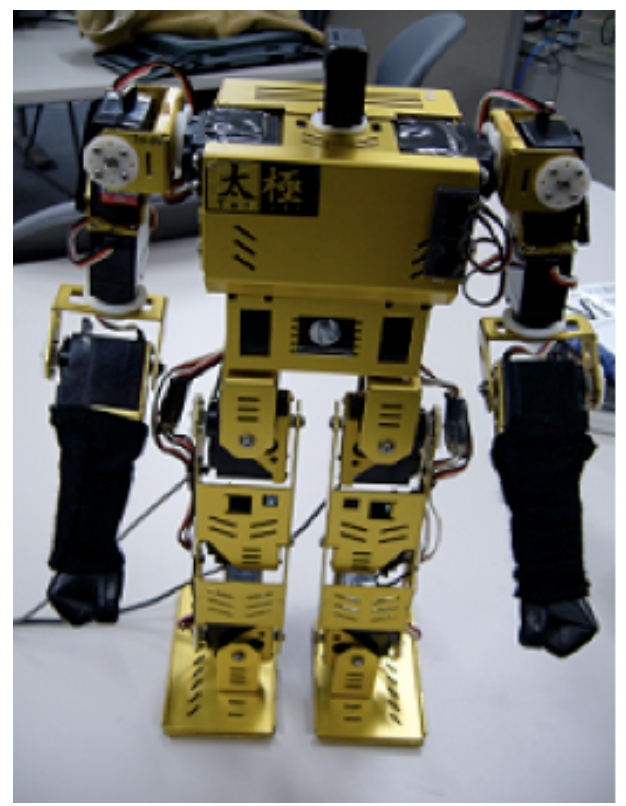

Fig. 1. Humanoid robot

Table 1. Specifications of humanoid robot

\begin{tabular}{cc}
\hline $\begin{array}{c}\text { Size/Weight } \\
\text { Degree of } \\
\text { flexibility }\end{array}$ & $34 \mathrm{~cm} / 1.7 \mathrm{~kg}$ \\
CPU & $22(12$ legs, 8 arms, 1 waist, 1 head $)$ \\
Motor & SH2/7047F \\
Battery & KO PDS-2144, FUTABA S3003, FUTABA \\
S3102, FUTABA S3103 \\
DC6V
\end{tabular}

We have carried out various kinds of studied using this prototype robot. We have developed a robot that can achieve Chinese exercise called Tai-chi [8]. Also we have proposed and developed a robot system that can achieve various kinds of dance motions [9][10]. Through these studied we have confirmed that this robot has a stability, flexibility, and sustainability to be used to various kind of applications. Thus we have adopted this robot for our study.

Figure 3 illustrates the software construction. The calculation of the commands necessary to move each motor is carried out each fifteen milliseconds and sent to each servo motor. The instructions to the robot from the PC are first analyzed and based 


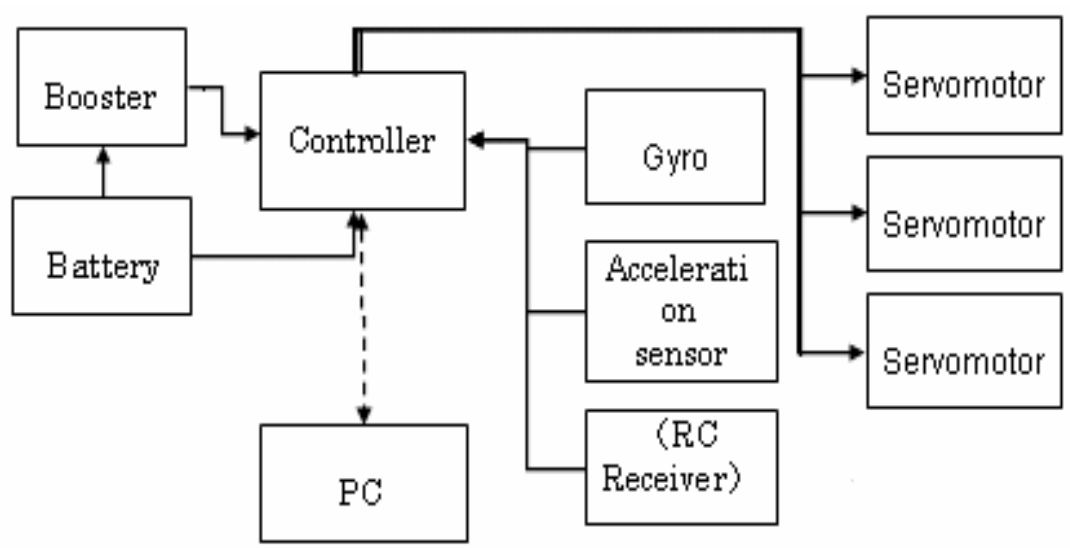

Fig. 2. Hardware construction of humanoid robot

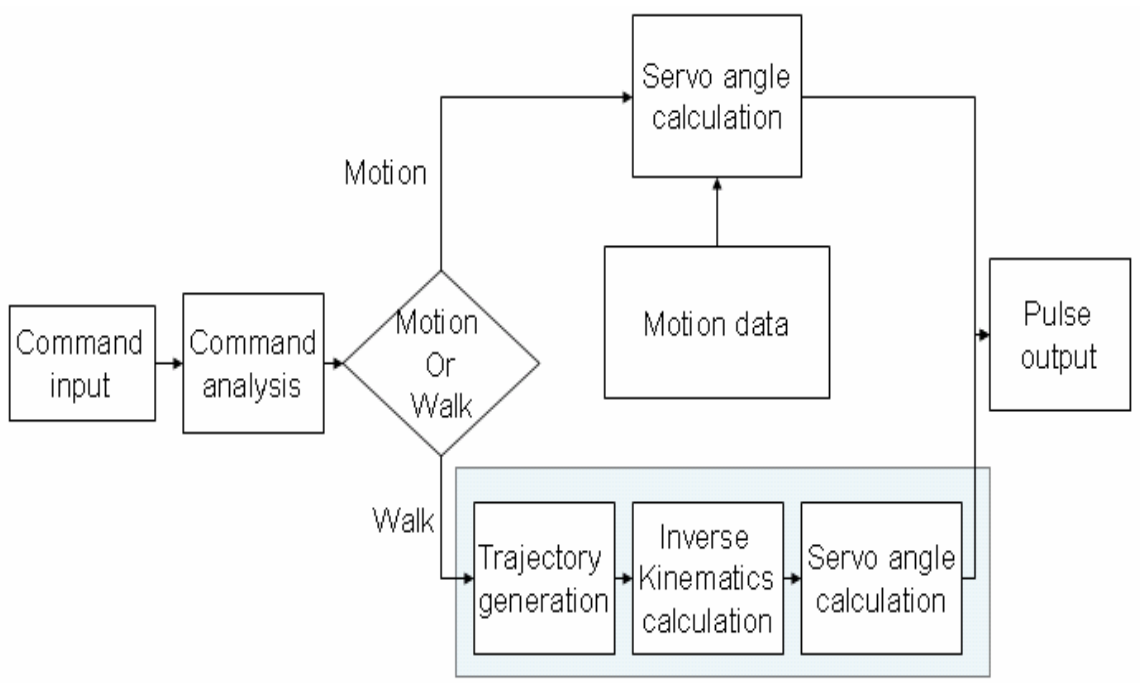

Fig. 3. Software construction of humanoid robot

on results go through one of two processes: one command for walking and other commands for other motions. For other commands, the motion data corresponding to the command is read from memory and the control data for each motor is calculated, and then the control data is sent to each servo motor. On the other hand, if the input command is a command for walking, then the real time calculation of the control data for each servo motor is carried out and sent to each servo motor. Calculation consists of three processes: trajectory generation calculation, inverse kinematics calculation, and servo motor angle calculation. In trajectory generation calculation, the position of each ankle studied by observing human walking motion is calculated every fifteen seconds. Then by inverse kinematics calculation the rotation angle of each foot joint is calculated for the same timing. Based on these calculations, finally the angle of 
each servo motor is calculated. Thus the rotation angle to be achieved for each motor is sent every fifteen milliseconds.

\section{Centaur Robot}

\subsection{Overview}

We developed a centaur robot based on the humanoid robot described in the previous section. We prepared two humanoid robots and used one as a front body. For another robot, we only used its lower body as a back of the centaur robot. Then we connected these two parts by a flat plastic board that functions as the shoulder part. Figure 4 shows the centaur robot's appearance.

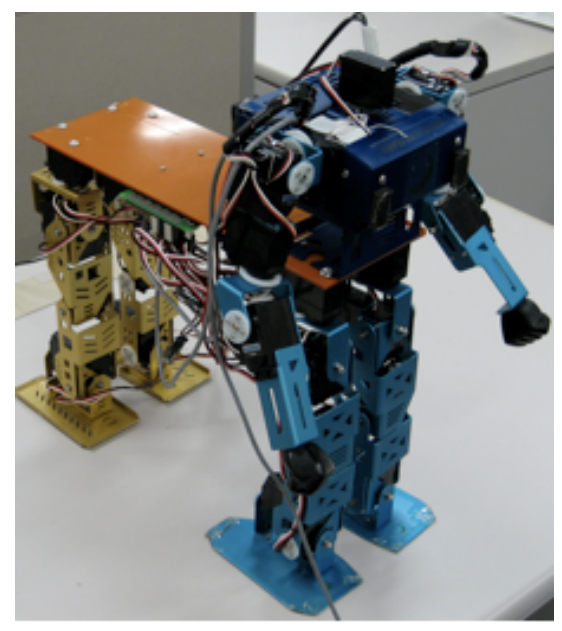

Fig. 4. Centaur robot

\subsection{Hardware Construction}

Here we explain the robot's hardware construction, as illustrated in Figure 5. Apparently for the front the hardware of the original humanoid robot was used, and for the back only the lower body was used. But a comparison of Figs. 3 and 5 shows that this robot's control structure is somewhat different from the original. Two controllers were used for complete control of the robot. One controls the servo motors required for upper body motions. The other controls the servo motors corresponding to the lower body. Since all the sensors are provided for the upper body, the controller corresponding to the upper body manages all sensor feedback. We adopted these two boards for several reasons. One, by using two boards, one of which controls the motions of the upper body and the lower body, it is possible to separately control the behaviors of the upper body as well as the lower body. For the power supply and battery, both controllers are connected to one battery. Also commands from PC are sent to both controllers. 


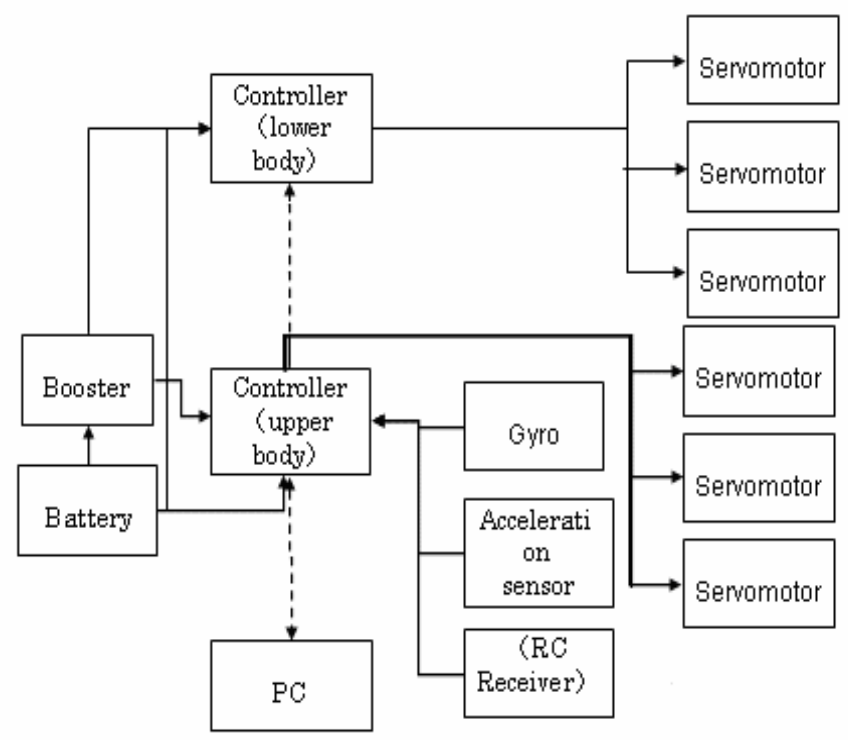

Fig. 5. Hardware construction of centaur robot

\subsection{Software Construction}

Next, we explain the robot's software construction, as illustrated in Figure 6. The software of the original humanoid controls both the upper and lower bodies together. For the centaur robot, we checked all the original robot's software and separated the software codes into two groups: one that controls the upper body and another that controls the lower body. Thus we reconstructed the whole software. For the upper body, it is unnecessary to carry out calculations for walking. When commands other than a walking command are sent from the PC, it retrieves motion data stored in the memory and sends the necessary rotation angle data to each servo motor. On the other hand software corresponding to the lower body must treat two types of commands as in the case of the original humanoid robot: a command for walking and other commands for additional motions. Also we adopted a method of inserting an arbitrary phase shift between the servo motor control of the front and back legs so that the robot can adopt the most adequate walking motions depending on the walking speed.

By adopting such basic software structure, robot control has the following merits:

(1) The upper and lower body motions can be controlled separately. So far all the motion data developed for achieving various types of humanoid robot motions must be developed to describe the whole body movement. Since the motions of the upper and lower bodies have been separated, we can separately develop two types of motions, and by combining these two types of data, we can generate various kinds of whole body movements for the robot. This idea can easily be applied to the original humanoid robot. 


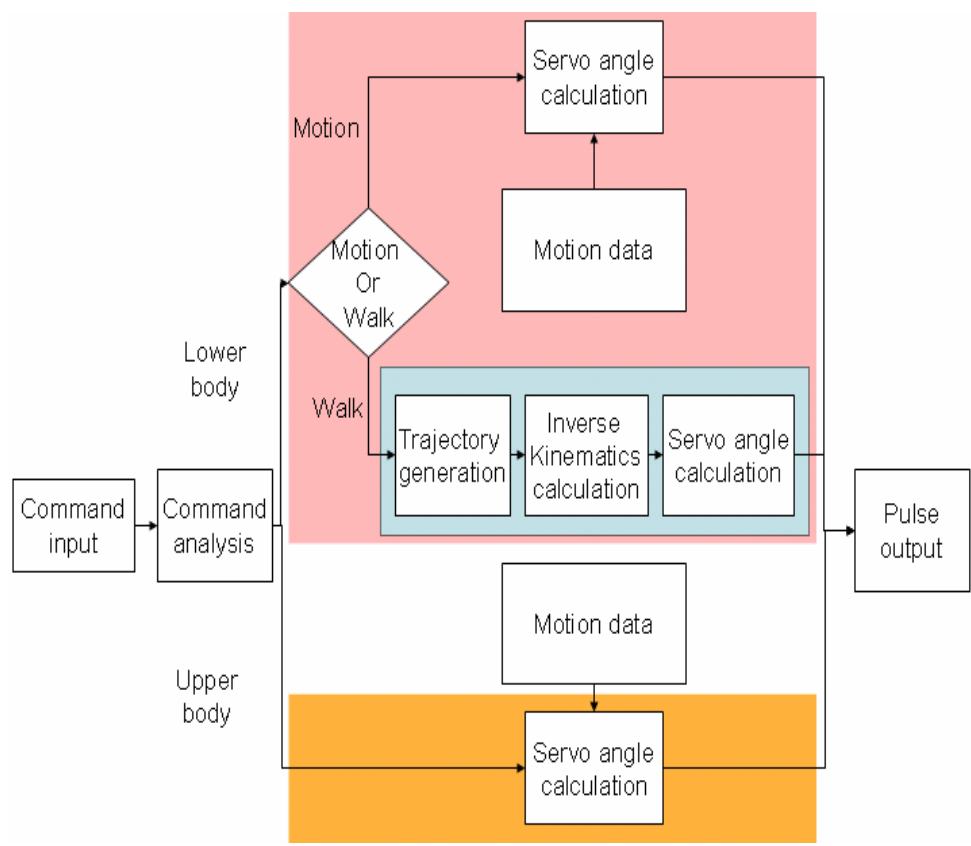

Fig. 6. Software construction of centaur robot

(2) The front and back body movements can be separately controlled. Although it seems natural to let the front lower body and back lower bodies perform identical motions, sometimes it is better to control the two bodies by different body motions. Especially in the case of walking and running motions there would be some differences between these two bodies. For example, for trot type walking there should be a $180^{\circ}$ phase shift between the front and the back legs. In the case of gallop running, the front legs and the back legs should move synchronously.

\subsection{Evaluation of the Robot}

We carried out several experiments to evaluate the motion capability of our centaur robot.

\section{(1) Walking capability}

We inserted a phase shift of 0 degree, 90 degree, and 180 degree between the walking motion cycle of the front and back legs. These walking styles correspond to those of animals such as "pace," "gallop," and "trot." We confirmed that the robot could move smoothly with almost the same speed by adopting each of these walking styles. Therefore for walking stability and speed all of the three walking styles perform the same capability.

As a next step, as we expect that one of the applications of this robot would to carry light load, we evaluated the walking stability from a point of carrying a load. 
For this we measured the tilt angle of the shoulder when it walks by fixing a gyro sensor on its shoulder and obtaining tilt data from it. We observed the time sequence data of the tilt angle five times for each walking style and averaged the data. Figures 7,8 , and 9 show the obtained data for a phase shift of 0 degree (pace), 90 degree (gallop), and 180 degree (trot).

Figures 7, 8, and 9 show that the tilt angle for pace walking style is larger than other two styles especially at a first step. Thus this waking style is not adequate for carrying a load such as a teacup or a newspaper. When comparing gallop and trot waling styles, although for the first step the tilt angles are almost the same, for the following steps trot walking style shows far better result. In this case of trot walking the front and back legs move in opposite modes. For example, when the front left leg moves forward, so does the back right leg. These results show that the tilt angle

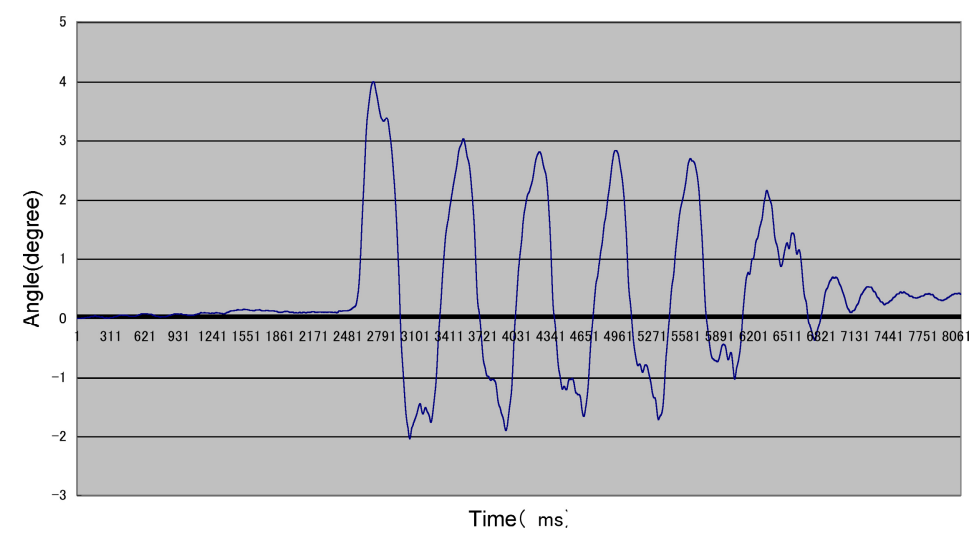

Fig. 7. Tilt angle for "pace" walking style

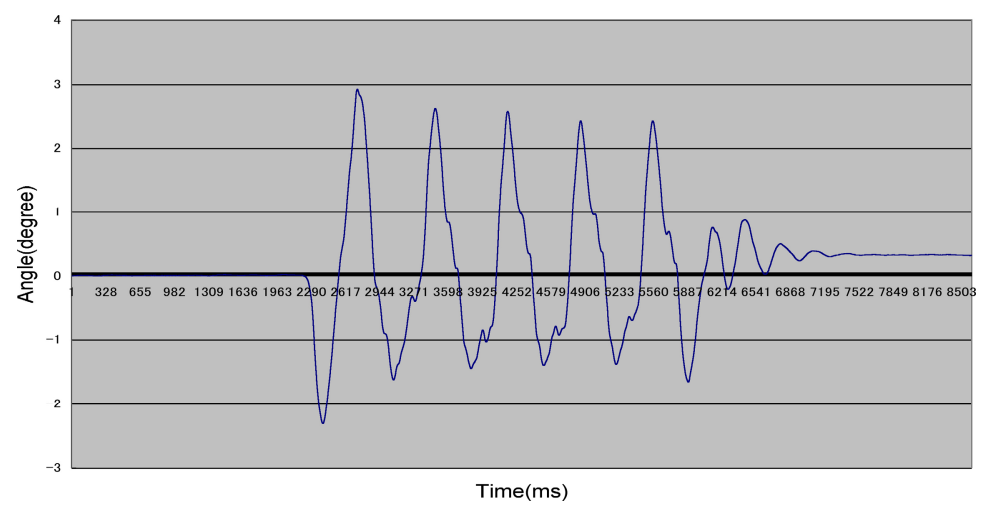

Fig. 8. Tilt angle for "gallop" walking style 


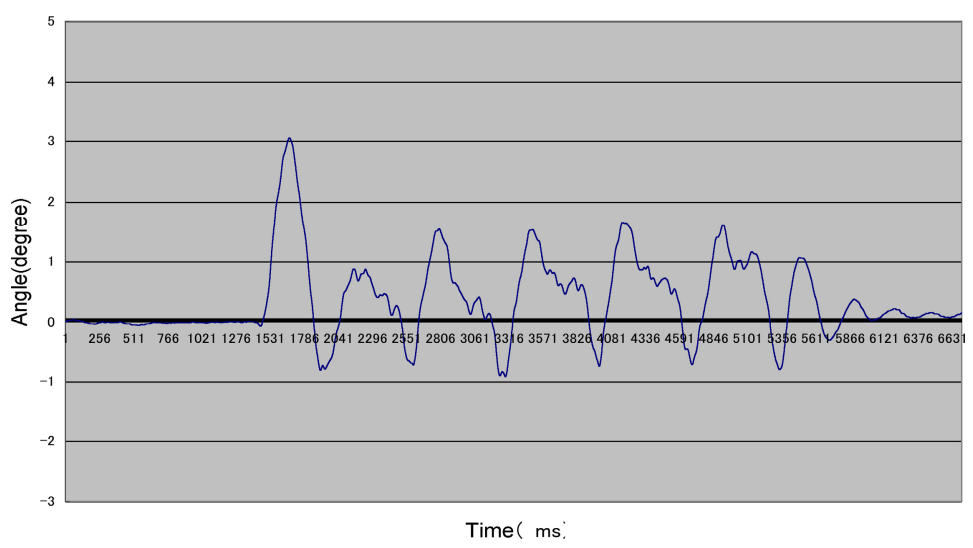

Fig. 9. Tilt angle for "trot" walking style

for trot walking style is lower than the other two and thus more stable when carrying a load. At the same time even in the case of trot, the first step causes a little bit large tilt angle. And this is the issue we have to improve in the further study.

(2) Capability for other motions

We developed various types of human-like motions for the original humanoid robot [8]. An interesting question is which of these motions could work well on the centaur robot. We tried to transfer the humanoid robot motions to this robot and found that most of the motions worked fairly well on this robot. On the other hand, motions including such postures as bending and twisting did not work well or needed modifications. One interesting future research theme is automatically transferring the humanoid robot motions to the motions of four-legged robot such as this robot.

\section{Conclusion}

In this paper we described the evaluation of walking motion stability of a robot we have proposed and developed. In the previous paper we proposed a new type of robot called "Centaur Robot" that is an integration of two types of robots: humanoid and four-legged. We adopted a humanoid robot with two legs and walking capability as a platform for this new robot. By integrating two of the humanoid robots we easily and successfully developed a centaur robot. In the previous paper we indicated that this robot has a function of stable walking. This time we carried out quantitative evaluation experiments to measure the stability of its waking motion. We confirmed that by inserting a phase shift of 0 degree, 90 degree and 180 degree between the front and back leg motions the robot can stably achieve pace, gallop, and trot walking motions. Then we evaluated these three walking styles from a point of tilt angle of its shoulder and found that the trot walking style is more stable than other styles. Since this robot has merits of both humanoid and four-legged robots, we are also going to evaluate its new capabilities that neither of the two type robots could achieve by themselves. 


\section{References}

1. http: //www.honda/co.jp/ASIMO/

2. Friedmann, M., Kiener, J., Petters, S., Thomas, D., Von Stryk, O., Sakakmoto, H.: Versatile, high-quality motions and behavior control of humanoid soccer robots. In: Workshop on Humanoid Soccer Robots of the 2006 IEEE International Conference on Humanoid Robots, pp. 9-16 (2006)

3. http://www.jp.aibo.com/

4. Golubovic, D., Li, B., Hu, H.: A Hybrid Software Platform for Sony AIBO Robots. In: Polani, D., Browning, B., Bonarini, A., Yoshida, K. (eds.) RoboCup 2003. LNCS (LNAI), vol. 3020, pp. 478-486. Springer, Heidelberg (2004)

5. Kerepesi, A., Kubinyi, E., Jonsson, G.K., Magnusson, M.S., Kiklosi, A.: Behavioural Comparison of Human-Animal (Dog) and Human-Robot (AIBO) Interactions. Behavioural Processes 73(1), 92-99 (2006)

6. Ishiguro, H., Ono, T., Imai, M., Kanda, T.: Development of an interactive humanoid robot "Robovie" -An interdisciplinary approach. In: Jarvis, R.A., Zelinsky, A. (eds.) Robotics Research, pp. 179-191. Springer, Heidelberg (2003)

7. Tsuda, S., Oda, Y., Shinozaki, K., Nakatsu, R.: Concept and Architecture of a Centaur Robot. In: Ma, L., Rauterberg, M., Nakatsu, R. (eds.) ICEC 2007. LNCS, vol. 4740, pp. 381388. Springer, Heidelberg (2007)

8. Wama, T., Higuchi, M., Sakamoto, H., Nakatsu, R.: Realization of Tai-chi Motion Using a Humanoid Robot. In: Rauterberg, M. (ed.) ICEC 2004. LNCS, vol. 3166, pp. 14-19. Springer, Heidelberg (2004)

9. Shinozaki, K., Oda, Y., Tsuda, S., Nakatsu, R., Iwatani, A.: Study of Dance Entertainment Using Robot. In: Pan, Z., Aylett, R.S., Diener, H., Jin, X., Göbel, S., Li, L. (eds.) Edutainment 2006. LNCS, vol. 3942, pp. 473-483. Springer, Heidelberg (2006)

10. Shinozaki, K., Iwatani, A., Nakatsu, R.: Concept and Construction of a Robot Dance System. The International Journal of Virtual Reality 6(3), 29-34 (2007) 